\title{
A Spitzer Spectrum of the Exoplanet HD 189733b
}

\section{Citation}

Grillmair, C. J., D. Charbonneau, A. Burrows, L. Armus, J. Stauffer, V. Meadows, J. Van Cleve, and D. Levine. 2007. "A Spitzer Spectrum of the Exoplanet HD 189733b." The Astrophysical Journal 658 (2): L115-18. https://doi.org/10.1086/513741.

\section{Permanent link}

http://nrs.harvard.edu/urn-3:HUL.InstRepos:41397453

\section{Terms of Use}

This article was downloaded from Harvard University's DASH repository, and is made available under the terms and conditions applicable to Other Posted Material, as set forth at http:// nrs.harvard.edu/urn-3:HUL.InstRepos:dash.current.terms-of-use\#LAA

\section{Share Your Story}

The Harvard community has made this article openly available.

Please share how this access benefits you. Submit a story.

\section{Accessibility}




\title{
A SPITZER SPECTRUM OF THE EXOPLANET HD 189733b
}

\author{
C. J. Grillmair, ${ }^{1}$ D. Charbonneau, ${ }^{2,3}$ A. Burrows,${ }^{4}$ L. Armus, ${ }^{1}$ J. Stauffer, \\ V. Meadows, ${ }^{1}$ J. Van Cleve, ${ }^{5}$ and D. Levine ${ }^{1}$ \\ Received 2007 January 19; accepted 2007 February 12; published 2007 February 23
}

\begin{abstract}
We report on the measurement of the 7.5-14.7 $\mu \mathrm{m}$ spectrum for the transiting extrasolar giant planet HD $189733 \mathrm{~b}$ using the Infrared Spectrograph on the Spitzer Space Telescope. Although the observations comprise only $12 \mathrm{hr}$ of telescope time, the continuum is well measured and has a flux ranging from 0.6 to $1.8 \mathrm{mJy}$ over the wavelength range, or $0.49 \% \pm 0.02 \%$ of the flux of the parent star. The variation in the measured fractional flux is very nearly flat over the entire wavelength range and shows no indication of significant absorption by water or methane, in contrast with the predictions of most atmospheric models. Models with strong day/night differences appear to be disfavored by the data, suggesting that heat redistribution to the night side of the planet is highly efficient.
\end{abstract}

Subject headings: binaries: eclipsing — planetary systems — stars: individual (HD 189733)

\section{INTRODUCTION}

HD 189733 is a nearby $\mathrm{K} 0 \mathrm{~V}$ star with a magnitude of $K=5.5$ and an expected flux of $250 \mathrm{mJy}$ at $10 \mu \mathrm{m}$. HD 189733 b was initially discovered in the ELODIE search for transiting exoplanets (Bouchy et al. 2005) and subsequently detected in existing Hipparcos data (Hébrard \& Lecavelier des Etangs 2006). In addition to HD 189733b, HD 189733 is orbited by an M dwarf at a distance of 216 AU (Bakos et al. 2006a). HD 189733b orbits its parent star in 2.2 days and has an estimated radius of $R_{p}=1.154 \pm 0.033 R_{\mathrm{J}}$ (Bakos et al. $2006 \mathrm{~b}$ ). From first to last contact, the duration of the secondary eclipse is $\approx 1.9 \mathrm{hr}$.

The planet-to-star surface area ratio for HD $189733 \mathrm{~b}$ is $\approx 0.024$ (Bakos et al. 2006b). Owing to an orbital radius of $0.031 \mathrm{AU}$, the upper atmospheric temperature of the planet is $\geq 1000 \mathrm{~K}$ (Fortney et al. 2006b). Together these factors yield an expected planet-to-star flux ratio that is almost a factor of 2 greater at $10 \mu \mathrm{m}$ than for either TrES-1 or HD 209458b, two other exoplanets whose thermal emission has recently been detected by Spitzer (Charbonneau et al. 2005; Deming et al. 2005). This expectation has been borne out by Deming et al. (2006) who used the Peak-up Imaging array portion of Spitzer's Infrared Spectrograph (IRS) to measure a secondary eclipse depth at $16 \mu \mathrm{m}$ of $0.551 \% \pm 0.030 \%$. This makes HD $189733 \mathrm{~b}$ a prime candidate for IRS spectroscopy and a more detailed characterization of its physical properties.

We briefly describe the observations in $\S 2$ and the analysis in $\S 3$. We discuss the spectrum of HD 189733b in $\S 4$.

\section{OBSERVATIONS}

HD 189733 was observed with the IRS on 2006 October 21 and November 21, as part of General Observer program 30473. In each case the star was observed for a $6 \mathrm{hr}$ span centered on

\footnotetext{
${ }^{1}$ Spitzer Science Center, Pasadena, CA 91125; carl@ipac.caltech.edu, lee@ipac.caltech.edu, stauffer@ipac.caltech.edu, vsm@ipac.caltech.edu, deblev@ipac.caltech.edu.

${ }^{2}$ Harvard-Smithsonian Center for Astrophysics, Cambridge, MA 02138; dcharbon@cfa.harvard.edu.

${ }^{3}$ Alfred P. Sloan Research Fellow.

${ }^{4}$ Steward Observatory, University of Arizona, Tucson, AZ 85721; burrows@ as.arizona.edu.

${ }^{5}$ Ball Aerospace and Technologies Corporation, Boulder, CO 80306; jvanclev@ ball.com.
}

the predicted time of secondary eclipse. All observations were made in first order $(\lambda=7.5-14.7 \mu \mathrm{m})$ using the Short-Low module, giving a spectral resolution of $\approx 80$. The performance and capabilities of the Short-Low module, which has the cleanest and most sensitive of the IRS detectors, is discussed in detail by Houck et al. (2004).

The October 21 observations were carried out in "staring" mode, wherein the telescope was periodically repointed to place the target alternately at two dither positions along the slit. Alternating between two slit positions provided some insurance against the possible large buildup of latent charge (see below). The observations were preceded by a high-accuracy peak-up approximately $3 \mathrm{hr}$ before secondary eclipse and were taken continuously through the out-of-eclipse, ingress, secondary eclipse, egress, and out-of-eclipse portions of HD 189733b's orbit. Thirty successive $14 \mathrm{~s}$ exposures were taken at each slit position before moving to the next dither position. A total of 30 telescope moves were commanded, yielding 450 independent integrations at each dither position over the course of $6 \mathrm{hr}$.

A preliminary analysis of the October 21 observations showed that charge latents were considerably more benign than initial estimates. An observing sequence scheduled for November 21 was consequently modified to place the target at the center of the Short-Low slit for the entire observing sequence. Small pointing errors associated with any commanded telescope movement (which contribute significantly to the noise budget) were thus avoided. Having thereby also eliminated telescope slew and settle times, we were able to fit a total of $95014 \mathrm{~s}$ exposures into the $6 \mathrm{hr}$ available.

\section{ANALYSIS}

Our analysis makes use of the two-dimensional Basic Calibrated Data products from the S14 version of the IRS pipeline. Using version 1.4 of the SPICE spectral extraction tool, we apply the optimal extraction feature to extract one-dimensional spectra for each of the 1850 exposures separately. We fix the center of each extraction window to take advantage of the pointing performance of the telescope and avoid adding noise associated with a measurement of the subpixel position of the star for each exposure.

For reasons that will become clear, we treat the integrations at the two dither positions on October 21 and the single-pointing integrations taken on November 21 as three separate sets 
of observations. In principle, it should be possible to simply co-add all integrations in each observation set taken during eclipse (starlight only) and subtract them from a co-addition of all spectra taken out of eclipse (star plus planet). In practice, due to the limited integration time available when the planet is in eclipse, several systematics can manifest themselves as either increased noise or spectral offsets.

Spitzer's stationary pointing performance is not perfect. Due to a small, cyclic heat source within the spacecraft structure, there is a periodic variation in alignment between the Star Tracker assembly and the telescope boresight. This leads to a pointing oscillation with a period of about an hour and a magnitude of $\sim 0.05^{\prime \prime}$ (Morales-Calderon et al. 2006). Although small, this oscillation has significant consequences for observations that require precision on the order of $0.1 \%$. In addition to variations in the total amount of light entering the slit, we are subject to significant signal modulation due to drifts across pixels with different (and poorly calibrated) sensitivities.

We analyze each wavelength bin as an independent time series and then combine the results to produce a final ratio spectrum. As an example, we show in Figure 1 the measured flux as a function of time at dither position 1 for the wavelength bin centered on $7.93 \mu \mathrm{m}$. Several characteristics that affect all wavelength bins to a greater or lesser degree are in evidence: (1) There is a rapid rise in the counts during the first 10 minutes of the sequence that is clearly related to latent charge buildup. (2) There is a longer term variation that is probably related to both the buildup and decay of latent charge, as well as to possible drifts in and out of the slit. (3) There is a cyclic behavior due to the pointing oscillations.

We have found that the buildup and decay of latent charge can vary greatly from one pixel to the next. Rather than trying to model the ensemble, short-term latent behavior for each wavelength bin, we simply exclude the first 10 minutes of data from our analysis. Since our final signal-to-noise ratio is determined primarily by data taken during secondary eclipse, the loss of the first 10 minutes of data has negligible consequences for the final result. We fit the longer term variation with a third-order polynomial and then divide it out to flatten the time series. We find this to be the minimum order necessary to reproduce the observed drifts while leaving the periodic variations largely unaffected.

Observations with the Infrared Array Camera indicate that Spitzer's pointing oscillation behaves rather more like a sawtooth than a sinusoid (Morales-Calderon et al. 2006). We indeed find that an asymmetric sawtooth best fits the cyclic time behavior in the data, and that the shape of the sawtooth is fairly consistent from one slit position to the next. We find that the phase and asymmetry of the flux modulation (which depend on both the telescope motion and the distribution of imperfectly calibrated pixels) are essentially constant for all wavelength bins (modulo a $180^{\circ}$ phase flip), and we adopt a fixed modulation pattern, with only the amplitude of the oscillation being a free parameter. The sawtooth that best fits the data for dither position 1 is shown overplotted on the drift-corrected data in the middle panel of Figure 1.

After dividing the data by the best-fitting periodic function, we fit the data using the ephemerides and the secondary eclipse profile of Bakos et al. (2006b). The uncertainty in the predicted time of the center of the secondary eclipse is 5.4 minutes assuming a circular orbit. The ephemerides we have adopted are consistent with updated values recently published by Winn et al. (2007). The timing uncertainty has been reduced to

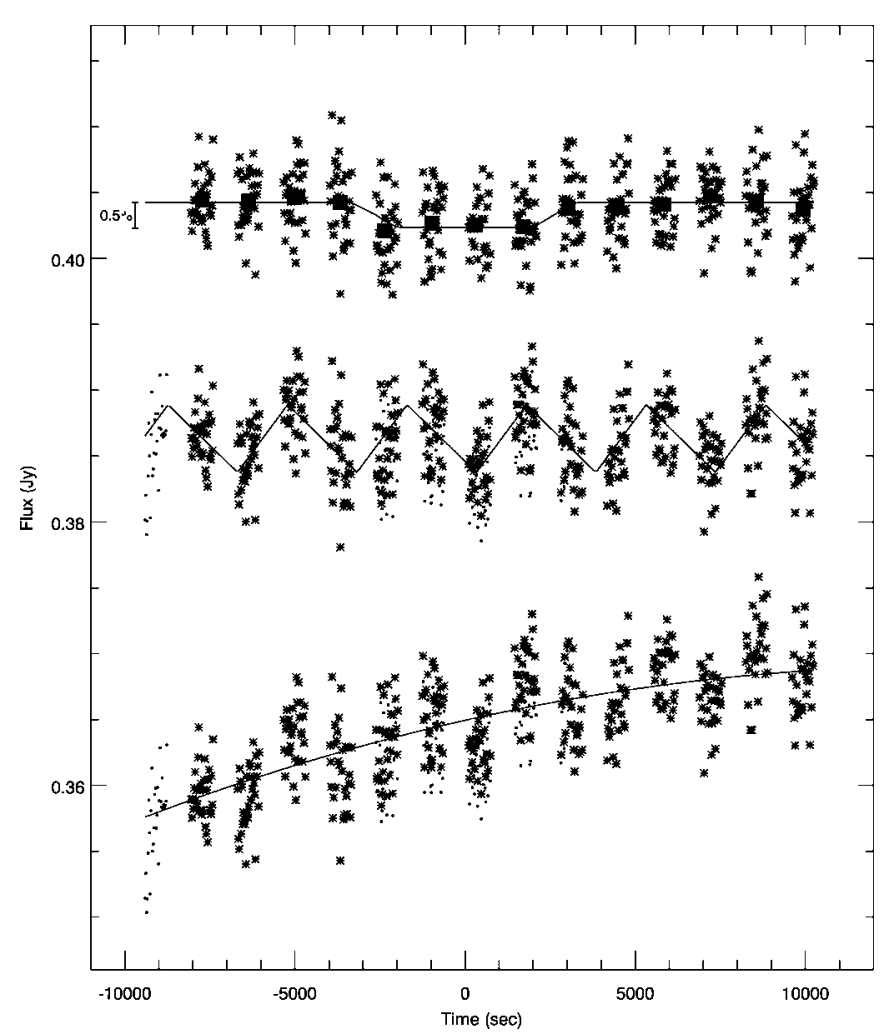

FIG. 1.-Best-fit solution for the planetary flux in the wavelength bin centered on $7.93 \mu \mathrm{m}$, showing from bottom to top the corrections applied. The flux measurements are shown as a function of time from secondary eclipse. The upper and lower data sets have been vertically offset for clarity. The filled circles in the bottom plot show the uncorrected flux as extracted using SPICE. The asterisks highlight data points actually used to (1) constrain the long-term drift, (2) fit the amplitude of the periodic function, and (3) fit the eclipse depth. To better constrain (1) and (2), the asterisked data have been corrected upward during secondary eclipse using the predicted light curve of Bakos et al. (2006b). The first 10 minutes of data are not used as they are generally affected by rapid latent charge buildup. The middle set of data points shows the effect of dividing by a third-order polynomial, and overplotted is the sawtooth function that best fits the pointing-induced oscillations. The upper set of data points has been corrected for long-term and periodic behavior, and the best-fitting light curve is shown overplotted. The filled squares are each a mean of 30 successive data points and serve simply as an aid in visualizing the quality of the fit.

0.5 minutes and is not a significant source of uncertainty in our analysis. In fitting the light curve, the two free parameters are the mean flux level outside of eclipse and the scaling of Bakos et al.'s light curve required to match the in-eclipse data. This allows us to use data taken during ingress and egress as well as during totality. We optimize the fit by varying the amplitude of the periodic function and minimizing $\chi^{2}$ during the in-eclipse portion of HD 189733b's orbit. Due to its greater extent, the mean level of the time series outside of eclipse is largely unaffected by changes in the periodic function, and can be independently measured to high precision.

To constrain the nonperiodic behavior of pixels in each wavelength bin over the course of $6 \mathrm{hr}$, we first divide the precorrection, in-eclipse data with the light curve of Bakos et al. (2006b). We find that simply fitting to all data with $t<-2500 \mathrm{~s}$ and $t>$ $2500 \mathrm{~s}$ is not sufficient to constrain long-term drifts during eclipse and adds noticeably to the noise in the final spectrum. The Bakos et al. (2006) light curve is initially scaled so that the upward correction at eclipse center is $0.5 \%$. We then iterate on the final solution for each wavelength bin by rescaling the correction based 


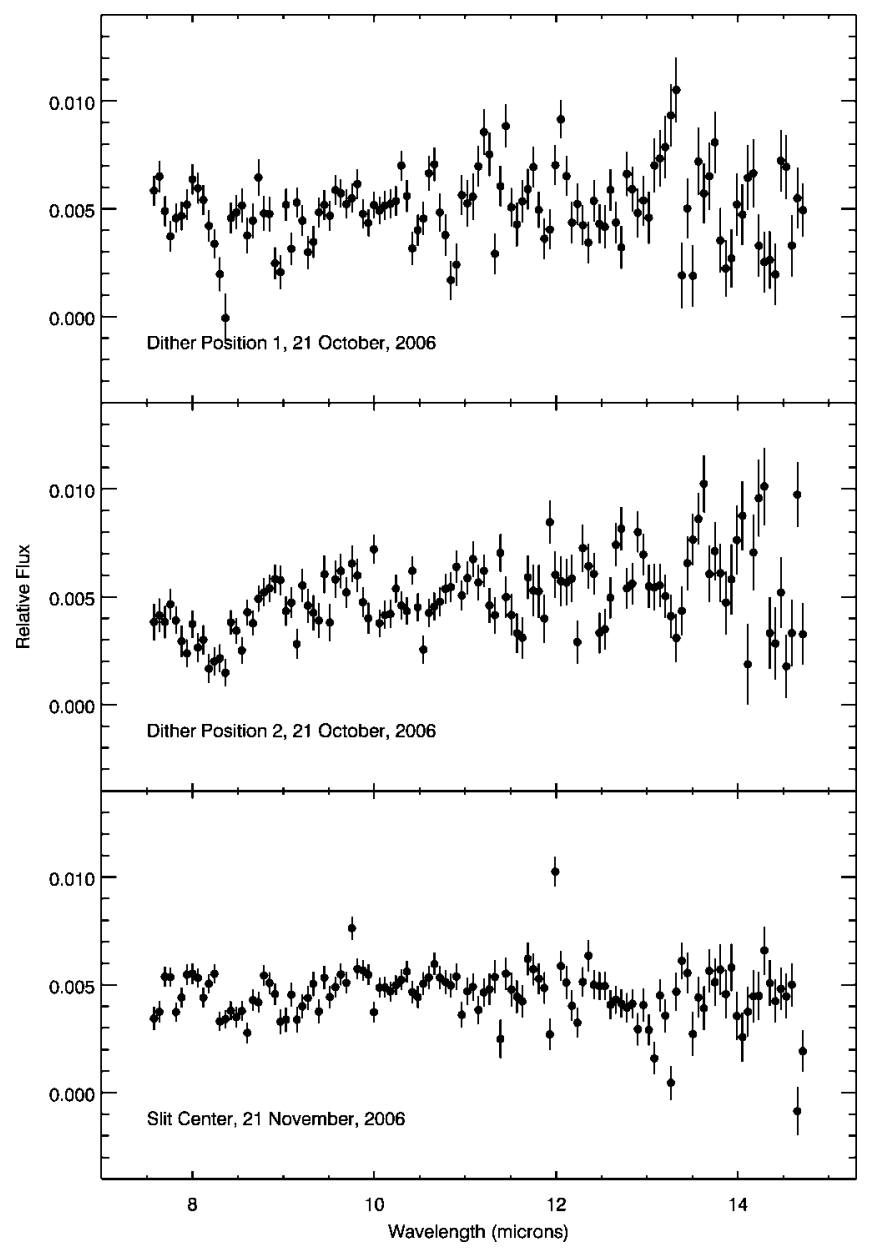

FIG. 2.-Flux of HD 189733b relative to that of HD 189733, as measured at three different slit positions. The spectra are unsmoothed, and the plotted uncertainties correspond to the formal fitting errors.

on the previous flux estimate. The solution converges rapidly in all cases, and we halt the calculation when the difference in computed eclipse depth between successive iterations becomes less than $0.002 \%$. Changing the initial scaling of the light curve by a factor of 2 up or down has no effect on the final spectrum.

Given a perfectly stable detector and platform, we would normally expect to be dominated by photon noise over the entire wavelength range for a star as bright as HD 189733. After making the corrections described above, the rms spread of the individual measurements about the best-fit light curve are indeed within $20 \%$ of the expected photon noise limit over most of the wavelength range. The rms spread in the individual measurements exceeds what one would expect just from photon noise by up to $50 \%$ in the regions 9-10, 11.5-12.5, and 13.7$14.5 \mu \mathrm{m}$ for both the October 21 and November 21 observations. Conversely, the regions 8-9, 10-11, and 12.5-13.5 $\mu \mathrm{m}$ show an rms spread that is consistent with pure photon statistics. This suggests that there are remaining, unmodeled temporal variations on the order of $0.5 \%$, due perhaps to fringe motions and subpixel variations in responsivity.

In Figure 2 we show the HD 189733b/HD 189733 flux ratios for each of the three separately analyzed data sets. The plotted uncertainties reflect only the light-curve fitting errors and are smallest for the November 21 observations by virtue of their greater number and continuous coverage through secondary eclipse.

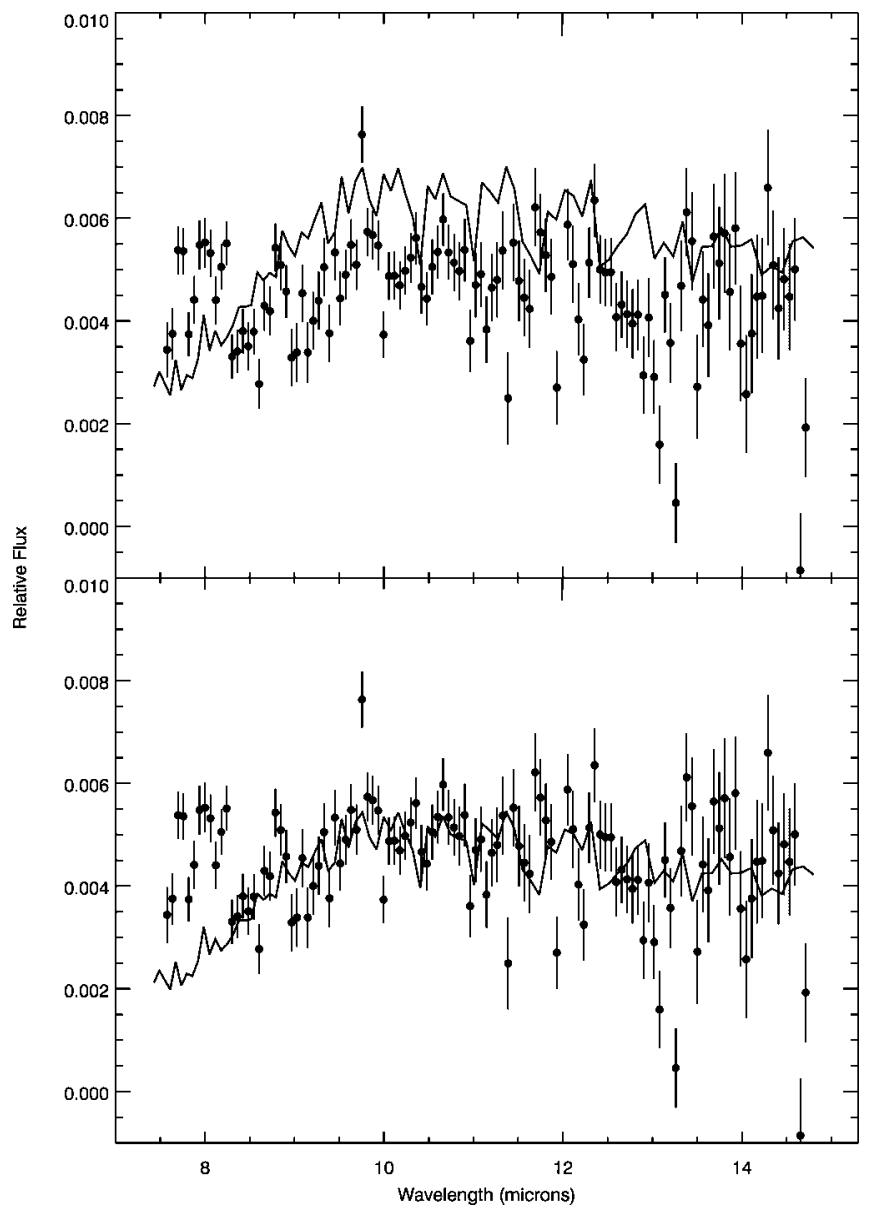

FIG. 3.-Comparison of the flux ratios measured at slit center (filled circles) with a model of HD 189733b from Burrows et al. (2006). The model shown is for superior conjunction, with no clouds and 50\% energy redistribution to the night side of the planet. The upper panel shows the model as published by Burrows et al. (2006), while in the lower panel the model has been scaled to match the data at $\lambda>9.5 \mu \mathrm{m}$. Intriguingly, the observed spectrum does not show the expected decrease in relative flux at short wavelengths due to increasing water opacity.

\section{DISCUSSION}

HD 189733b's continuum is well measured and, multiplying the relative fluxes in Figure 2 by the observed spectrum of HD 189733 , we obtain absolute fluxes ranging from $1.8 \mathrm{mJy}$ at $7.8 \mu \mathrm{m}$ to $0.5 \mathrm{mJy}$ at $14.7 \mu \mathrm{m}$. Our measurement is consistent with the $660 \mu \mathrm{Jy}$ integrated flux measurement at $16 \mu \mathrm{m}$ by Deming et al. (2006). Comparing the three spectra to one another, the signal-to-noise ratio per resolution element ranges from about 15 at $10 \mu \mathrm{m}$ to about 6 at $14.5 \mu \mathrm{m}$.

The flux ratios in Figure 2 are essentially flat; the emission spectrum of HD $189733 \mathrm{~b}$ over the wavelength range 7.5$14.7 \mu \mathrm{m}$ is evidently consistent with that of a blackbody. The integrated flux ratios are $0.50 \%, 0.51 \%$, and $0.46 \%$, respectively, yielding a mean of $0.49 \% \pm 0.02 \%$. Although the mean relative flux is in good agreement, the spectrum taken at dither position 1 appears somewhat problematic, with a number of peaks and troughs that are not seen at the other slit positions. Of the three, the spectrum taken at slit center is clearly the highest quality measurement and demonstrates the utility of keeping the telescope as motionless as possible for observations of this type. While there are interesting similarities between the spectra taken at dither position 2 and at slit center, the 
differences are sufficiently large and numerous that we cannot identify individual absorption or emission features with any confidence.

In Figure 3 we compare the relative flux spectrum measured at slit center with a model of HD 189733b from Burrows et al. (2006). The model shown is for superior conjunction, with no clouds and $50 \%$ energy redistribution to the night side of the planet. The mean levels for the model and the observations are quite similar. Redward of $10 \mu \mathrm{m}$, where the flux ratio is predicted to be fairly flat, the model predicts a mean relative flux that is about $20 \%$ higher than that of the observations. Near superior conjunction, less atmospheric energy redistribution would push the relative planet/star flux still higher. In contrast to the findings of Harrington et al. (2006) for $v$ And b, it appears that strong day/night differences on HD 189733b are disfavored by the data.

Blueward of $8.2 \mu \mathrm{m}$, the model predicts a quite significant drop in the relative flux due to water absorption. In contrast, the observed flux ratios continue unabated to the blue end of the spectrum. This difference is significant; scaling the Burrows et al. (2006) model to match the data beyond $9.5 \mu \mathrm{m}$ and summing over all bins blueward of $8.2 \mu \mathrm{m}$, the model lies $\approx 9 \sigma$ below the data. The measured flux at the blue end is not the product of a few bad measurements but is reflected separately in each of the three individual spectra and two independent analyses. It is also seen in simple subtractions of combined out-of-eclipse and in-eclipse spectra.

A reduced planet-to-star ratio blueward of $10 \mu \mathrm{m}$ due to water opacity is a near-universal prediction in published models of hot Jupiters to date (e.g., Fortney et al. 2005; Barman et al. 2005; Seager et al. 2005; Burrows et al. 2006), and hence the absence of this signature from our data is intriguing. Simply reducing the amount of water in the models may not, by itself, bring them into agreement with the data. For example, our observations also appear to disagree with the low $\mathrm{H}_{2} \mathrm{O}$, high carbon-to-oxygen ratio spectrum computed for HD 209458 b by Seager et al. (2005). In this case a similar drop in the flux blueward of $9 \mu \mathrm{m}$ is expected due to methane absorption. We note that Fortney et al. (2006a) have recently considered dynamical models of hot Jupiter atmospheres (Cooper \& Showman 2006) to predict emergent spectra as a function of orbital phase and have shown that spectral features in dayside spectra may be suppressed due to an isothermal pressure-temperature profile. A more thorough analysis of the consequences of this spectrum for models of giant planet atmospheres is forthcoming.

The detection of HD 189733b's spectral continuum, accomplished with only $12 \mathrm{hr}$ of Spitzer time, is a remarkable demonstration of the capability and utility of the Spitzer Space Telescope and the Infrared Spectrograph. Integrating over many eclipses, we can expect substantial increases in spectral signalto-noise ratio, broader wavelength coverage, and the detection of atmospheric constituents for both HD 189733b and other transiting extrasolar giant planets. While Spitzer's cryogen lasts, we can look forward to an exciting era of quantitative, spectral characterization of extrasolar planets, and ultimately a deeper understanding of planetary atmospheres under strong irradiation.

We are grateful to an anonymous referee for several suggestions that significantly improved the analysis and presentation of this work. This work is based on observations made with the Spitzer Space Telescope, which is operated by the Jet Propulsion Laboratory, California Institute of Technology, under a contract with NASA. Support for this work was provided by NASA through an award issued by JPL/Caltech. This study was supported in part by NASA grant NNGO4GL22G and through the NASA Astrobiology Institute under Cooperative Agreement CAN-02-OSS-02 issued through the Office of Space Science.

\section{REFERENCES}

Bakos, G. A., Pal, A., Latham, D. W., Noyes, R. W., \& Stefanik, R. P. 2006a, ApJ, 641, L57

Bakos, G. A., et al. 2006b, ApJ, 650, 1160

Barman, T. S., Hauschildt, P. H., \& Allard, F. 2005, ApJ, 632, 1132

Bouchy, F., et al. 2005, A\&A, 444, L15

Burrows, A., Sudarsky, D., \& Hubeny, I. 2006, ApJ, 650, 1140

Charbonneau, D., et al. 2005, ApJ, 626, 523

Cooper, C. S., \& Showman, A. P. 2006, ApJ, 649, 1048

Deming, D., Harrington, J., Seager, S., \& Richardson, L. J. 2006, ApJ, 644, 560

Deming, D., Seager, S., Richardson, L. J., \& Harrington, J. 2005, Nature, 434, 740

Fortney, J. J., Cooper, C. S., Showman, A. P., Marley, M. S., \& Freedman, R. S. 2006a, ApJ, 652, 746
Fortney, J. J., Marley, M. S., Lodders, K., Saumon, D., \& Freedman, R. 2005, ApJ, 627, L69

Fortney, J. J., Saumon, D., Marley, M. S., Lodders, K., \& Freedman, R. S. 2006b, ApJ, 642, 495

Harrington, J., Hansen, B. M., Luszcz, S. H., Seager, S., Deming, D., Menou, K., Cho, J. Y.-K., \& Richardson, L. J. 2006, Science, 314, 623

Hébrard, G., \& Lecavelier des Etangs, A. 2006, A\&A, 445, 341

Houck, J. R., et al. 2004, ApJS, 154, 18

Morales-Calderon, M., et al. 2006, ApJ, 653, 1454

Seager, S., Richardson, L. J., Hansen, B. M. S., Menou, K., Cho, J. Y.-K., \& Deming, D. 2005, ApJ, 632, 1122

Winn, J. N., et al. 2007, AJ, in press (astro-ph/061224) 